\title{
A study to compare the diagnostic accuracy of GeneXpert MTB/RIF/assay and its comparison with liquid culture in clinically suspected cases of genital tuberculosis attending outpatient department of tertiary center
}

\author{
Anu Pathak, Divya Yadav, Urvashi Verma, Yogita Gautam*
}

Department of Obstetrics and Gynecology, S. N. Medical College, Agra, Uttar Pradesh, India

Received: 17 March 2020

Revised: 22 June 2020

Accepted: 30 June 2020

\author{
*Correspondence: \\ Dr. Yogita Gautam, \\ E-mail: yogitagautam100@gmail.com
}

Copyright: (C) the author(s), publisher and licensee Medip Academy. This is an open-access article distributed under the terms of the Creative Commons Attribution Non-Commercial License, which permits unrestricted non-commercial use, distribution, and reproduction in any medium, provided the original work is properly cited.

\begin{abstract}
Background: Tuberculosis is a major health issue globally despite a declining trend in mortality with effective diagnosis and treatment, an estimated 10.4 million persons developing active TB each year with 1.33 million deaths. Objective of this study was to evaluate role of GeneXpert MTB/RIF/assay in diagnosis of female genital tuberculosis in suspected cases of tuberculosis.

Methods: It was a cross sectional study done in department of obstetrics and gynecology in S. N. Medical college Agra for a period of 2 year (July 2017 to October 2019). 70 cases were selected from OPD of department of obstetrics and gynecology, S. N. Medical College Agra who met the inclusion and exclusion criteria after taking proper consent. In all selected cases endometrial biopsy sample was taken using endometrial biopsy curette in premenstrual period. All samples of endometrial biopsy were taken under all aseptic precaution from both corneal ends, anterior and posterior wall and lower part of uterus using endometrial biopsy curette and sample was collected in two separate sterile vials having normal saline and was sent for GeneXpert MTB/RIF/assay and liquid culture simultaneously.

Results: Out of total 70 clinically suspected cases of female genital tuberculosis in between 20-45 years of age cough with expectoration $94 \%$ was the most common respiratory symptom followed by fever $81 \%$, weight loss $56 \%$ and anorexia 54\%. Prevalence of genital tuberculosis in active pulmonary tuberculosis patients was 30\%. Irregular menstruation, vaginal discharge and pelvic pain were present in $69 \%, 60 \%$ and $52 \%$ patients respectively.

Conclusions: The overall sensitivity of CBNAAT was $22 \%$ and specificity was $77 \%$. The overall sensitivity of liquid culture was $28 \%$ and specificity was $71 \%$.
\end{abstract}

Keywords: Endometrial biopsy, Genital tuberculosis, GeneXpert, Liquid culture

\section{INTRODUCTION}

Tuberculosis is a major health issue globally despite a declining trend in mortality with effective diagnosis and treatment, an estimated 10.4 million persons developing active TB each year with 1.33 million deaths. ${ }^{1}$ TB is one of the top three infectious killing disease in the world. HIV/AIDS kills 3 million people each year, TB kill 2 million and malaria kills 1 million. ${ }^{2}$
Most (85\%) TB deaths occur in developing countries, especially Asia (55\%) and Africa (30\%) with most cases from $20-45$ years age group. ${ }^{3}$ Genital TB usually occur secondary to $\mathrm{TB}$ in other sites (primarily lungs) the spread is generally through hematogenous or lymphatic routes.

Multidrug resistant (MDR) and extensive drug resistance (XDR) are a matter of real concern. In 2015 nearly 
500,000 women died from TB and among them $28 \%$ had human immunodeficiency virus co-infection. ${ }^{4}$

The possibility of FGTB should be considered in patients with chronic PID not responding to standard antibiotic treatment, unexplained infertility or in women with irregular menstrual cycle or postmenopausal bleeding and persistent vaginal discharge (where genital neoplasia's have been excluded). ${ }^{5}$

Apart from infertility other clinical presentations include oligomenorrhea, amenorrhea, menorrhagia, abdominal pain, dyspareunia, and dysmenorrhea, abdominal swelling, pelvic pain, postcoital bleeding, abnormal vaginal discharge not responding to treatment. ${ }^{6}$ The fallopian tubes are involved in $90-100 \%$ cases, endometrium is involved in $50-80 \%$ cases, ovaries in 20 $30 \%$ cases, and cervix is involved in $5-15 \%$ cases of genital TB. ${ }^{7}$

Now the most recent test used to diagnose TB includes GeneXpert MTB/RIF assay which is a new test for tuberculosis which works on molecular level to identify MTB. It has a dual advantage i.e. it can detect MTB bacilli in the given specimen and it can also detect resistance to one of the most common drugs, rifampicin.

The World Health Organization (WHO), guideline gave a strong and conditional recommendation for use of GeneXpert as the initial diagnostic test in adults and children who have MDR TB or HIV associated TB as the initial diagnostic test based on the Cepheid GeneXpert system, a platform for rapid and simple to use nucleic acid amplification test (NAAT). ${ }^{8}$

The GeneXpert MTB/RIF is a cartridge based fully automated nucleic acid amplification test, automated diagnostic test that can identify mycobacterium tuberculosis (MTB), DNA and resistance to rifampicin (RIF) by nucleic acid amplification test (NAAT). It purifies concentrates, amplifies (by rapid, real time PCR) and identifies targeted nucleic acid sequences in the TB genome and provide results from processed endometrial biopsy samples in less than 2 hours, with minimal handson technique. It was co-developed by the laboratory of Professor David Alland at the University of Medicine and Dentistry of New Jersey (UMDNJ). ${ }^{9}$

The BACTEC-460 system (Becton-Dickinson microbiology systems, Sparks, Md.) was the first semiautomated liquid medium system introduced for rapid detection of mycobacterial growth, and after about 20 years of successful use in many clinical laboratories it can be considered the one most frequently in standard use among such system. ${ }^{10}$

The advantages of liquid culture include its sensitivity, identification of mycobacterium species and ability to perform phenotypic drug susceptibility tests (DSTs) and genotyping for further molecular epidemiology studies.
The disadvantage of culture methods is the time needed for the growth of mycobacteria. Liquid cultures require at least 9-10 days for positive results and six weeks for being considered negative and in LJ medium cultures, the minimum time-to-positivity is $4-8$ weeks. ${ }^{11}$

\section{METHODS}

The study was a prospective comparative study conducted in the department of obstetrics and gynecology S. N. Medical College, Agra and department of TB and chest, S. N. Medical College, Agra during the study period of 2 years (July 2017 to Oct 2019), 70 cases were selected from gynecology OPD of department of obstetrics and gynecology, S. N. Medical College Agra who met the inclusion and exclusion criteria after taking proper consent.

\section{Inclusion criteria}

- Unexplained infertility

- Infertile cases with tubal pathology

- PID not responding to routine antibiotic treatment

- Unexplained menorrhagia

- Unexplained secondary infertility

- Chronic pelvic pain

- Oligomenorrhea.

\section{Exclusion criteria}

- Pregnant females

- Suspicion of malignancy

- Unmarried females

- Known cases of tuberculosis

- Patient on ATT.

Detailed history including age, socioeconomic status, history suggestive of risk factors were taken. A complete general physical examination, local examination and bimanual examination was done. All the clinically suspected females of GTB were called in their premenstrual period for endometrial biopsy. All routine investigations were done.

In all selected cases endometrial biopsy sample was taken using endometrial biopsy curette in premenstrual period and sample was sent in two sterile vials separately both for liquid culture and GeneXpert MTB/RIF/assay.

All samples of endometrial biopsy was taken under all aseptic precaution from both corneal ends, anterior and posterior wall and lower part of uterus using endometrial biopsy curette and sample was collected in two separate sterile vials having normal saline and was sent for GeneXpert MTB/RIF/assay and liquid culture simultaneously. The results of GeneXpert came after 24 hours and those who were tested positive for the test were started on ATT accordingly based whether the resistance for rifampicin was present or not. 


\section{RESULTS}

Out of all 70 female patients with active pulmonary tuberculosis $20(28.57 \%)$ were sputum positive and 50 $(71.43 \%)$ were sputum negative. Mean age of the patients was 29 \pm 7.68 . Minimum age of the patient was 20 years and maximum age of the patient was 45 years. Maximum number of patients was $35(50 \%)$ in 20-30 years age group followed by $29(41.43 \%)$ in $31-40$ years age group. The sputum smear positivity rate was $28.57 \%$. The sputum positive patients were high in all groups and equally distributed among all age groups.

Table 1: Age distribution of sputum positive cases.

\begin{tabular}{|lllllll|}
\hline \multirow{2}{*}{ Age distribution (in year) } & \multicolumn{2}{l}{ Sputum positive } & \multicolumn{2}{l|}{ Sputum negative } & Total & \\
\cline { 2 - 7 } & No. & Percentage & No. & Percentage & No. & Percentage \\
\hline $20-30$ & 11 & $55 \%$ & 24 & $48 \%$ & 35 & $50 \%$ \\
\hline $31-40$ & 8 & $40 \%$ & 21 & $42 \%$ & 29 & $41.43 \%$ \\
\hline$>40$ & 1 & $5 \%$ & 5 & $10 \%$ & 6 & $8.57 \%$ \\
\hline Total & 20 & $28.57 \%$ & 50 & $71.43 \%$ & 70 & $100 \%$ \\
\hline
\end{tabular}

Table 2: Cases with close contact of tuberculosis.

\begin{tabular}{|lll|}
\hline History of close contact & No. of cases & Percentage \\
\hline Present & 11 & $16 \%$ \\
\hline Absent & 59 & $84 \%$ \\
\hline Total & 70 & $100 \%$ \\
\hline
\end{tabular}

Table 3: Associated gynecological complaints.

\begin{tabular}{|lll|}
\hline Symptoms & No. of cases & Percentage \\
\hline Vaginal discharge & 42 & $60 \%$ \\
\hline Pelvic pain & 37 & $52 \%$ \\
\hline Normal menstruation & 22 & $31 \%$ \\
\hline Amenorrhea & 18 & $26 \%$ \\
\hline Hypomenorrhea & 13 & $19 \%$ \\
\hline Menorrhagia & 17 & $24 \%$ \\
\hline Infertility & 42 & $60 \%$ \\
\hline
\end{tabular}

Most of this study patients had almost equal distribution of rural and urban. Rural was (51\%), that was slightly more than urban (49\%). In this study group DM was most commonly $(7 \%)$ associated medical disorder followed by COPD in $6 \%$ cases (Table 1 ).

In this study group $16 \%$ of patients had history of close contact in family and in $84 \%$ history of close contact was absent (Table 2).
Among the gynecological symptoms irregular menstruation, vaginal discharge and pelvic pain were present in $69 \%, 60 \%$ and $52 \%$ of this study patients respectively. Normal menstruation was in $31 \%$ patients. Out of $69 \%$ patients with irregular menstruation, amenorrhea in $26 \%$ and menorrhagia in $24 \%$ were more common symptoms (Table 3).

In total 70 study patients, 12 patients were CBNAAT positive and 20 patients were HPE positive. Out of total (12) CBNAAT positive genital tuberculosis patients 11 patients were positive on HPE and 1 patient was negative. Out of total (20) patients positive on HPE, 11 were CBNAAT positive and 9 were negative (Table 4). In total 70 study patient overall sensitivity and specificity of CBNAAT was found to be $22 \%$ and $77 \%$ respectively and of liquid culture was found to be $28 \%$ and $71 \%$ respectively (Table 5).

Out of 70 patients 47 infertile patients CBNAAT was found to be negative in 35 patients and in 12 infertile patients CBNAAT was found positive (25.53\%) and CBNAAT was found negative in 35 patients $(74.47 \%)$. Out of 70 patients 47 infertile patients' liquid culture was found to be negative in 30 patients and in 17 infertile patients' liquid culture was found positive (36.17\%) and liquid culture was found negative in 30 patients $(63.83 \%)$ (Figure 1).

Table 4: Genital TB patients confirmed with HPE and CBNAAT.

\begin{tabular}{|lllllll|} 
& \multicolumn{2}{l}{ CBNAAT positive } & \multicolumn{2}{l|}{ CBNAA } & & Total \\
& No. & Percentage & No. & Percentage & No. & Percentage \\
\hline HPE positive & 11 & $55 \%$ & 9 & $45 \%$ & 20 & $100 \%$ \\
\hline HPE negative & 1 & $2 \%$ & 49 & $98 \%$ & 50 & $100 \%$ \\
\hline Total & 12 & $17.1 \%$ & 58 & $82.9 \%$ & 70 & $100 \%$ \\
\hline
\end{tabular}


Table 5: Overall sensitivity and specificity of CBNAAT and liquid culture in infertile patients.

\begin{tabular}{|lll|}
\hline Overall & CBNAAT & Liquid culture \\
\hline Sensitivity & $22 \%$ & $28 \%$ \\
\hline Specificity & $77 \%$ & $71 \%$ \\
\hline
\end{tabular}

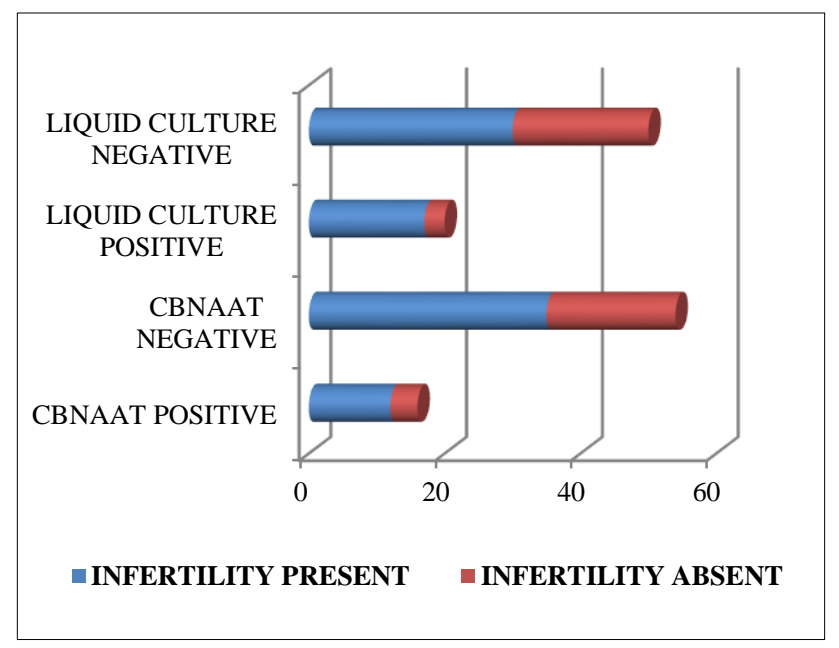

Figure 1: Genital tuberculosis diagnosed by CBNAAT and Liquid culture with infertility

\section{DISCUSSION}

This prospective cross-sectional study was carried out in department of obstetrics and gynecology in S. N. Medical College Agra during the period from July 2017 to October 2019.

In this study, authors investigated the female patients with clinical suspicion of genital tuberculosis using newer diagnostic methods namely cartridge based nucleic acid amplification test (CBNAAT) (GeneXpert MTB/RIF/assay).

Purpose of this study was to evaluate the females with clinical suspicion of genital tuberculosis for genital involvement and to find out the prevalence and various presentations of the same.

CBNAAT is the modern diagnostic technique, which gives reliable and quick results, and though it is not a gold standard test, it is supposed to be very close to it.

Authors observed the mean age of the patients was $29 \pm 7.68$ years (range 20-45). The sputum positive patients were high in all groups and equally distributed among all age groups.

Authors had almost equal distribution of females from rural $(51 \%)$ and urban $(49 \%)$ areas.

History of close contact in family was present in $16 \%$ of female patients in this study group.
It is mostly associated with comorbidities like diabetes mellitus (7\%), COPD (6\%), the most common associated symptom with FGTB is cough with expectoration (94\%) and fever $(81 \%)$, weight loss $(56 \%)$.

In this study irregular menstruation was the most common gynecological complaint followed by infertility, vaginal discharge and pelvic pain; in $69 \%, 60 \%, 60 \%$ and $52 \%$ cases respectively. ${ }^{7}$ Out of $68 \%$ patients with irregular menstruation, amenorrhea $26 \%$ and menorrhagia $24 \%$ were more common symptoms followed by hypomenorrhea $19 \%$.

In total 70 study patients, 12 patients were CBNAAT positive and 20 patients were HPE positive. Out of total 12 patients CBNAAT positive genital tuberculosis patients 11 patients were positive on HPE and 1 patient was negative. While out of total 20 patients positive on HPE, 11 were CBNAAT positive and 9 were negative.

In this study total 70 patients having clinical suspicion of female genital tuberculosis were enrolled and then endometrial biopsy sample was sent for CBNAAT and liquid culture; out of these 12 patients were reported positive on CBNAAT alone and 20 patients were positive on liquid culture; 21 patients had either findings suggestive of genital tuberculosis on ultrasonography.

In total 70 study patient overall sensitivity and specificity of CBNAAT was found to be $22 \%$ and $77 \%$ respectively and of liquid culture was found to be $28 \%$ and $71 \%$ respectively.

\section{CONCLUSION}

Genital tuberculosis continues to be a common disorder in women. GTB is now undergoing a worrying recrudescence. So, the disease needs renewed interest. Early diagnosis and appropriate treatment not only relieve the symptoms but there are more chances of fertility. New tools in the diagnostic armamentarium are urgently required for the rapid diagnosis of GTB and monitoring of GTB treatments, and to gain new insights into pathogenesis.

The GeneXpert MTB/RIF/assay is a rapid molecular assay that can be used close to the point of care by operators with minimal technical expertise, enabling diagnosis of TB and simultaneous assessment of rifampicin resistance to be completed within 2 hours.

Moreover, this can be accomplished using unprocessed sputum samples as well as clinical specimens from extrapulmonary sites.

The main advantages of the test are, for diagnosis, reliability when compared to sputum microscopy and the speed of getting the result when compared with the culture test whereas liquid culture takes 42 days for results to come. 
In this study authors found the sensitivity and specificity of GeneXpert to be $22 \%$ and $77 \%$ respectively as compared to liquid culture which has $28 \%$ and $71 \%$ respectively.

So, as to conclude authors can say that GeneXpert is a rapid and accurate diagnostic test for genital tuberculosis and to assess sensitivity to rifampicin. However, this is a small-scale study and large-scale studies should be done including a large number of patients with clinical suspicion of female genital tuberculosis for early diagnosis and management.

\section{Funding: No funding sources}

Conflict of interest: None declared

Ethical approval: The study was approved by the Institutional Ethics Committee

\section{REFERENCES}

1. Angeline G, Bella D, Natrajan M. Genital tuberculosis in females. Indian $\mathrm{J}$ Med Res. 2017;145:425-36.

2. Hershkovitz I, Donoghue HD, Minnikin DE, Bersa GS, Lee OYC, Gernaey AM, et al. Detection and molecular characterization of 9000-year-old mycobacterium complex from a noethilic settlement in the eastern Mditerranean. PLoS PMF. 2008;2:3426.

3. World Health Organization, global tuberculosis report: Geneva: WHO; 2019. Available at: https://www.who.int/tb/publications/global_report/en 1. Accessed on $12^{\text {th }}$ February 2020.

4. Sharma JB, Current diagnosis and management of female genital tuberculosis. J Obstet Gynaecol India. 2015;65:362-71.

5. Varma TR. Genital tuberculosis and subsequent fertility. Int J Gynecol Obstet. 1991;35:1-11.
6. Singh N, Sumana G, Genital tuberculosis: a leading cause for infertility in women seeking assisted conception in North India. Arch Gynecol Obstet. 2008;278:325-7.

7. Rothschild BM, Martin LD, Lev G, Bercovier H, Bar-Gal GK, Greenblatt CL, et al. Mycobacterium tuberculosis complex DNA from an Extinct Bison Dated 17,000 years before the present. Clin Infec Dis. 2001;33:5-11.

8. World Health Organization Xpert MTB/RIF assay for diagnosis of pulmonary and extra-pulmonary TB in adults and children - policy update. EHO/RIF/TB/2013.16. Geneva, Switzerland; WHO; 2013.

9. Banada PP, Koshy R, Alland D. Detection of mycobacterium tuberculosis in blood by use of the Xpert MTB/RIF assay. J Clin Microbiol. 2013;51(7):2317-22.

10. Daly JW, Monif GRG. Infectious diseases in obestatrics and gynaecology. Philadelphia Harper Row. 1982;2:301.

11. Norbis L, Alagna R, Tortoli E, Codecasa LR, Migliori GB, Cirillo DM. Challenges and perspectives in the diagnosis of extrapulmonary tuberculosis. Expert Rev Anti Infect Ther. 2014;12:633-47.

Cite this article as: Pathak A, Yadav D, Verma U, Gautam Y. A study to compare the diagnostic accuracy of GeneXpert MTB/RIF/assay and its comparison with liquid culture in clinically suspected cases of genital tuberculosis attending outpatient department of tertiary center. Int J Reprod Contracept Obstet Gynecol 2020;9:3345-9. 